\title{
Consulta farmacéutica: intoxicación por gemfibrozilo
}

\author{
Nerea Acuña Elvira \\ Doctora en Ciencias de la Salud. Farmacéutica comunitaria en Arrecife (Lanzarote).
}

\section{PALABRAS CLAVE}

Intoxicación por gemfibrozilo, protocolo normalizado de trabajo, servicio de seguimiento farmacoterapéutico

\section{ABREVIATURAS}

SFT: seguimiento

farmacoterapéutico

PNT: procedimiento

normalizado de trabajo

RAM: reacciones adversas al

medicamento

RNM: resultados negativos de

la medicación

\section{KEYWORDS}

Gemfibrozil poisoning, standardized work protocol, pharmacotherapeutic monitoring service

\section{RESUMEN}

Paciente de 46 años diagnosticada de depresión, hipertiroidismo e hipertrigliceridemia. En el último mes y medio ha tenido que acudir al servicio de urgencias del hospital en varias ocasiones por presentar cuadros diarreicos importantes acompañados de náuseas y malestar general. Los médicos le diagnosticaron gastroenteritis, administraron suero intravenoso con antieméticos y pautaron paracetamol $1 \mathrm{~g}$ cada 8 horas.

En la propia consulta, el marido solicita le dispensemos Trialmin ${ }^{\circledR} 600 \mathrm{mg}$, que su esposa "tiene prescrito para el tiroides" y del que toma 6 comprimidos diarios.

Detectamos un resultado negativo de la medicación por error en la pauta e indicación del medicamento para la hipertrigliceridemia. Intervenimos explicándole que Tirodril ${ }^{\circledR}$ es el medicamento que le han prescrito para el hipertiroidismo y no Trial$\min ^{\circledR}$, que está indicado en hipertrigliceridemias. Se deriva al servicio de urgencias con un informe por escrito de la situación dirigido al médico.

\section{Pharmaceutical consultation: gemfibrozil poisoning}

\section{ABSTRACT}

Forty-six-year-old patient diagnosed with depression, hyperthyroidism and hypertriglyceridemia. Over the last month and a half, she has had to go to the emergency service several times due to major diarrhea symptoms, nausea and malaise. The doctors diagnosed gastroenteritis, administered intravenous fluid with antiemetics and prescribed paracetamol $1 \mathrm{~g}$ every 8 hours.

During the consultation, her husband asked to be given Trialmin ${ }^{\circledR} 600 \mathrm{mg}$, which had been "prescribed for [his wife's] thyroid" at a dose 6 pills per day.

We detected a negative drug result stemming from a prescription error and the drug's indicated use for hypertriglyceridemia. We acted by explaining that Tirodril ${ }^{\circledR}$ is the drug that had been prescribed for hyperthyroidism, rather than Trialmin ${ }^{\circledR}$, which is indicated for hypertriglyceridemia. The patient is directed to the emergency service with a written report for the doctor.

\section{Presentación}

Se trata de una paciente de 46 años diagnosticada de depresión, hipertiroidismo e hipertrigliceridemia. En el último mes y medio ha tenido que acudir al servicio de urgencias del hospital en al menos tres ocasiones por presentar cuadros diarreicos importantes acompañados de náuseas y malestar general. En estas visitas los médicos diagnosticaron gastroenteritis, administraron suero intravenoso, con antieméticos y pautaron paracetamol $1 \mathrm{~g}$ cada 8 horas.
En este caso es el marido de la paciente el que acude a la farmacia y consulta porque está preocupado por el estado de salud de su esposa. La consulta se centra en si creemos que es posible que haya tenido gastroenteritis vírica o bacteriana de forma tan seguida y si pensamos que debería acudir a un especialista en digestivo. Al ser la primera vez que acude a nuestra farmacia le indicamos que tenemos un servicio de seguimiento farmacoterapéutico (SFT) en el que podemos estudiar todos los problemas de salud y los medicamentos con el fin de comprobar el uso necesario, seguro y efectivo de cada uno de ellos.

En la propia consulta, el marido nos solicita que le dispensemos el medicamento Trialmin ${ }^{\circledR} 600$ mg que su esposa tiene prescrito, ante esta situación intentamos obtener más información, siguiendo el procedimiento normalizado de trabajo (PNT) de dispensación [1] (figura 1) para comprobar que la dispensación de dicho medicamento se realiza de 


\section{FARMACIA ARGANA}

\section{DISPENSACIÓN ACTIVA CON PRESCRIPCIÓN}

Abuerre R, García-Delgado P, Maurndi MD, Arrebola C, Gastelurrutia MA, Martínez F. Diseño y pilotaje de un proceso estructurado para el servicio de dispensación de medicamentos. Aten Prim. 2015;47(1):7-14

¿Es para usted el medicamento o para alguien a su cuidado?

Sí: se continúa el PNT

No: se procede a la venta

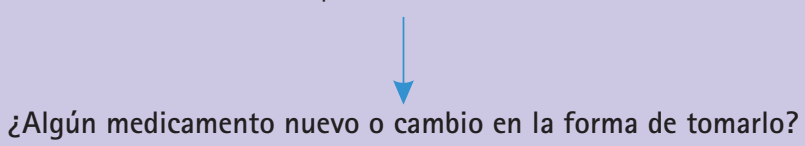

Si: se procede para ese medicamento a realizar el PNT de primera dispensación No: se procede para ese medicamento a realizar el PNT de dispensación repetida

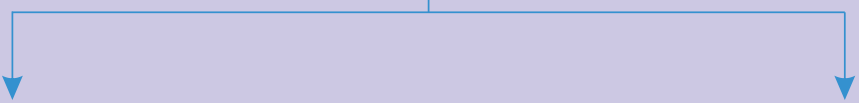

PNT primera dispensación

¿Tiene alergia a algún medicamento?

Si (a alguno de los que se van a dispensar): salida del PNT de dispensación No: continúa el PNT

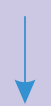

Dar IPM $\stackrel{\text { No conoce }}{\longleftarrow}$ ¿Para qué toma/utiliza el medicamento? Conoce Dar IPM No conoce ¿Cómo toma/utiliza el medicamento? Conoce

Dar IPM No conoce ¿Hasta cuándo tiene que tomar/ utilizar el medicamento?

$$
\text { Conoce }
$$

Dar IPM $\stackrel{\text { No conoce }}{\longleftarrow}$ ¿Cómo sabe si el medicamento le hace efecto?

\section{PNT dispensación repetida}

¿Considera usted que controla/mejora su problema de salud?

No: (existe percepción de inefectividad del tratamiento)

Sí: se pasa a evaluar la percepción de inseguridad

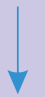

¿Ha notado usted algún problema de salud desde que toma el medicamento?

Si: (existe percepción de inseguridad del tratamiento)

- No: continuar el PNT

PNT: protocolo normalizado de trabajo

IPM: información personalizada del medicamento

AF: atención farmacéutica

¿Tiene otros problemas de salud (o situación fisiológica especial)?

Sí: ver contraindicaciones y/o duplicidades y/o teratogenia No: continuar con el PNT

\section{Sí: ver interacciones}

No: continuar con el PNT
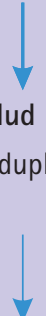

Entrega o no del medicamento

Derivación si procede al médico/profesional sanitario/otro servicio de $\mathrm{AF}$

Figura 1 Procedimiento normalizado de trabajo de dispensación 
manera que se garantice un uso adecuado de los medicamentos dispensados. De las respuestas del cuidador deducimos que no sabe para qué es el medicamento, porque nos dice que es el que su esposa toma para un hipertiroidismo que le han detectado hace poco más de dos meses. Le informamos que ese medicamento no es para esa indicación y vemos que es otro medicamento del plan de tratamiento (Tirodril ${ }^{\circledR}$ ) el que la paciente tiene prescrito para ese problema de salud. Indicamos al cuidador que tiene otro medicamento que sí que es para el hipertiroidismo pero que no es Trialmin $^{\circledR}$ sino Tirodril ${ }^{\circledR}$. Procedemos a la dispensación de Tirodril ${ }^{\circledR}$ porque está seguro de que el medicamento que su esposa necesita es el del tiroides y le indicamos su modo de empleo. Ofrecemos derivar al servicio de seguimiento farmacoterapéutico para hacer un estudio exhaustivo de los medicamentos y ver si encontramos la causa de la diarrea, pero para ello debe acudir la paciente.

Media hora más tarde, el marido vuelve enfadado porque su esposa lo ha hecho volver a la farmacia ya que el medicamento que ella "necesita" no es Tirodril ${ }^{\circledR}$ sino Trialmin $^{\circledR}$, que según ella es el del tiroides.

Solicitamos hablar por teléfono con la paciente, a lo que su marido accede, le preguntamos sobre la medicación que toma y nos indica, entre otras, que Trialmin ${ }^{\circledR}$ lo toma para el hipertiriodismo desde hace 2 meses, y que toma una dosis diaria de 6 comprimidos de $600 \mathrm{mg}$.

\section{Evaluación}

Ante esta situación abrimos un episodio de seguimiento farmacoterapéutico y consultamos en el BotPlus las fichas técnicas de ambos medicamentos [2].

Las reacciones adversas (RAM) más frecuentes de gemfibrozilo son gastrointestinales, observadas en más del 10\% de los pacientes. Aunque a las dosis adecuadas no suele provocar la interrupción del tratamiento, en este caso parece tratarse de un problema de intoxicación por confusión con la pauta terapéutica que tiene prescrita del medicamento antitiroideo, Tirodril ${ }^{\circledR}$ (Tiamazol $5 \mathrm{mg}$ ) (6 comprimidos diarios), ya que la dosis habitual de gemfibrozilo $600 \mathrm{mg}$ es 2 comprimidos diarios, por lo que se está administrando el triple de lo indicado [2]. Deducimos pues un resultado negativo de la medicación (RNM) de inseguridad de Trialmin ${ }^{\circledR}$ por toma de una dosis excesiva [3].

\section{Intervención}

Informamos a la paciente que lo más probable es que sus episodios gastrointestinales sean debidos a una sobredosis de gemfibrozilo, y le explicamos que es Tirodril ${ }^{\circledR}$ el medicamento que le han prescrito para el hipertiroidismo y no Trialmin ${ }^{\circledR}$, que está indicado en hipertrigliceridemias. La derivamos al servicio de urgencias del hospital con un informe por escrito de la situación dirigido al médico.

\section{Resultado}

La paciente es ingresada y el diagnóstico es intoxicación por gemfibrozilo. Su estancia en el hospital fue de 7 días en los que se realizó una monitorización clínica de gemfibrozilo. Se suspendió este medicamento y comenzó a tomar correctamente Tirodril $^{\circledR}$ consiguiendo así una mejoría de los síntomas relacionados con el hipertiroidismo.

La paciente acudió a la farmacia para ser incluida en el servicio de SFT y así quedarse más tranquila al conseguir la correcta utilización de todos los medicamentos prescritos.

\section{Discusión}

De la valoración final de este caso clínico se resaltan tres aspectos fundamentales en la práctica farmacéutica diaria: la importancia de realizar una correcta dispensación de los medicamentos que asegure su uso adecuado, acorde con los principios de necesidad, efectividad y seguridad; la evidente necesidad de avanzar hacia una farmacia más asistencial en la que se disponga de servicios de atención farmacéutica o servicios profesionales farmacéuticos con el fin de mejorar el proceso de uso de los medicamentos y por tanto sus resultados; y se vuelve a poner de manifiesto la inaplazable necesidad de que los farmacéuticos podamos tener acceso a la historia clínica de los pacientes y disponer de un servicio de comunicación inmediata con los médicos y/u otros profesionales de la salud [4]. Además, se aprecia en este caso la importancia de evitar similitudes [5] en los nombres de los medicamentos comercializados, ya que pueden derivar en confusiones que pongan en riesgo la salud de los pacientes.

Probablemente, si la paciente hubiese recibido un auténtico servicio de dispensación, se hubiese beneficiado de una actuación profesional y con ello evitado la intoxicación y los costes clínicos y económicos derivados de ella.

\section{Referencias bibliográficas}

1. Abaurre R, García-Delgado P, Maurandi MD, Arrebola C, Gastelurrutia MA, Martínez Martínez F. Diseño y pilotaje de un proceso estructurado para el servicio de dispensación de medicamentos. Aten Primaria. 2015; 47(1):7-14. https://doi. org/10.1016/j.aprim.2014.01.005

2. Consejo General de Colegios Oficiales de Farmacéuticos. Bot Plus Web [Base de datos en internet] 2017. [Consultado el 21/01/2017]. Disponible en: https:// botplusweb.portalfarma.com/Documentos/2015/7/22/89102.pdf

3. Foro de Atención Farmacéutica en Farmacia Comunitaria. Guía práctica para los Servicios de Atención Farmacéutica en la Farmacia Comunitaria. Madrid: Consejo General de Colegios Oficiales de Farmacéuticos; 2010.

4. García-Delgado Morente A. Importancia de compartir la historia clínica entre los miembros del equipo de salud. Farmacéuticos Comunitarios. 2016; 8(Supl.1).

5. Otero López MJ, Martín Muñoz R, Sánchez Barba M, Abad Sazatornil R, Andreu Crespo A, Arteta Jiménez M, et al, en representación del Grupo de Trabajo RML. Elaboración de una relación de nombres de medicamentos similares en los que se recomienda utilizar letras mayúsculas resaltadas. Farm Hosp. 2011; 35:225-235. https:// doi.org/10.1016/j.farma.2011.06.004 